\title{
An Efficient Bond-adaptive approach for Finite-temperature Open Quantum Dynamics using the one-site Time-Dependent Variational Principle for Matrix Product States
}

\author{
Angus J. Dunnett ${ }^{1}$ and Alex W. Chin ${ }^{1}$ \\ ${ }^{1}$ Sorbonne Université, CNRS, Institut des NanoSciences de Paris, 4 place Jussieu, 75252 Paris, France
}

\begin{abstract}
Recent tensor network techniques for simulating system-environment wavefunctions have provided profound insights into non-Markovian dissipation and decoherence in open quantum systems. Here, we propose a dynamically adaptive one-site Time-Dependent-Variational-Principle (A1TDVP) method for matrix product states in which local bond dimensions grow to capture developing systembath entanglement. This avoids the need for multiple convergence runs w.r.t. bond dimensions and the unfavourable local Hilbert space scaling of two-site methods. A1TDVP is thus ideally suited for open quantum dynamics in finite-temperature bosonic environments, as the initial states typically have low bond dimension but require very large local physical dimensions. We demonstrate this with simulations of non-equilibrium heat flows through a qubit spin, finding a 30x and 10x speed-up over 2TDVP and 1TDVP, respectively.
\end{abstract}

\section{INTRODUCTION}

Irreversible phenomena such as relaxation and decoherence critically determine how physical and biochemical systems process free energy and quantum information at the nanoscale $6 / 7 / 13 / 47 / 61$. However, over the short spatio-temporal scales (fs-ns, nm) at which such nonequilibrium processes may occur, simple Markovian descriptions of open quantum systems (OQS) will typically fail. Instead, the key physics emerges from the growth and death of many body quantum correlations between the system and the external variables of its environment(s). Understanding how these extended quantum correlations and memory effects impact the performance of functional materials will be key for the optimization and design of efficient, few-quanta machines ${ }^{2}$, as well as suggesting new routes for avoiding deleterious environmental effects in quantum technologies ${ }^{1}$.

Describing how dissipation emerges from this complexity requires advanced, non-perturbative numerical techniques $3132|35| 53|54| 57 / 60 \mid 61$, and the use of tensor network states (TNS) have recently proved particularly effective for this problem $43|44| 46 / 50$. TNS provide highly efficient representations of large, correlated quantum states and are widely used to study non-perturbative

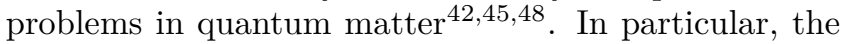
class of TNS known as matrix product states (MPS) are especially powerful for 1D systems with short-range couplings, and are thus ideally suited to the very wide range of system-environment Hamiltonians that can be mapped onto simple chain-like models (see Refs. $\frac{12146 / 56}{}$ ). Strikingly, a recent result of Tamascelli et al. allows access to finite-temperature (mixed state) effects from pure system-environment wavefunctions $\sqrt{56}$, opening TNS methods to real-world applications 20 .

However, the numerical resources required for fi- nite temperature simulations can grow rapidly over time ${ }^{19 \mid 20}$, necessitating efficient techniques for evolving the MPS. The time-dependent variational principle (TDVP) applied to MPS22/25|26/37/49, has established itself as a powerful numerical method for time evolving


over the long established time evolving block decimation (TEBD) algorithm is the fact that the only input it requires is a matrix product operator (MPO) representation of the Hamiltonian, meaning that it can be applied to generic Hamiltonians ${ }^{30}$, potentially with long range interactions, like a 'black-box'.

Unlike practically all other MPS algorithms which control the growth of bond dimensions by performing a compression after each evolution step, the evolution generated by TDVP is confined to the smooth manifold of MPS with fixed bond dimension $D$ (see Fig. 1). The TDVP attempts to find the MPS $\psi(t,\{A\})$ built from a set of matrices $\{A\}$ with bond dimensions $D$ which best approximates the actual time-evolved wavefunction. The set of MPS for full-ranked $\{A\}$ of fixed dimensions is a smooth manifold $\mathcal{M}$ on which this optimization can be performed 25 . The solution for a system with a Hamiltonian $\hat{H}(\hbar=1)$ is found to be

$$
\frac{d}{d t}|\psi(t,\{A\})\rangle=-i \hat{\mathcal{P}}_{T_{|\psi(t)\rangle, \mathcal{M}}} \hat{H}|\psi(t,\{A\})\rangle,
$$

where $\hat{\mathcal{P}}_{T_{|\psi(t)\rangle, \mathcal{M}}}$ is the orthogonal projector onto the tangent space of $\mathcal{M}$ at the point $|\psi(t,\{A\})\rangle$. The introduction of this projector results in a projection error $\epsilon_{\mathcal{P}}{ }^{31}$, due to the fact that the exact state will in general become highly entangled, and cannot be faithfully represented by a 1 D MPS ansatz $\mid \Psi(t,\{A\}\rangle$ in which the entanglement will be bounded by $\sim \log (D)$. On the other hand, Eq. (1) can be integrated exactly to within a time step error ${ }^{\underline{Z 0}}$, avoiding the truncation errors as- 
sociated with other time-evolution schemes $\frac{45}{4}$. The geometric properties of TDVP also include attractive features such as automatic norm conservation and Riemannian optimization 26127. Perhaps most interestingly in the context of open quantum system is its ability to accurately predict the apparent long-time 'thermalization' dynamics of observables associated with local degrees of freedom 23134 .

On the other hand, the fixed nature of MPS bond dimensions under TDVP is a major inconvenience whenever entanglement is spatially or temporally inhomogeneous. This is a particular problem in OQS problems where we are normally interested in the dynamics of an initial system-environment state with an exact $D=1$ product state structure. As entanglement then grows, much larger bond dimensions will be needed to describe the state, which, for fixed- $D$ TDVP dynamics, means that one must embed the initial, low-entanglement MPS in a much larger manifold to capture the later dynamics. Thus, at short-to-intermediate times, the bond dimensions will be much larger than those actually required by the physics, and multiple costly runs with varying bond dimensions are needed to converge the results. Moreover, as the Tamascelli theorem states that many open system problems have environments initially in the vacuum state ${ }^{56}$, (non-interacting) bath excitations are created and destroyed uniquely at the systemenvironment bond, and thus spread in a 'light cone' centered on the system site ${ }^{55}$. Using a large, uniform bond dimension for even the most 'distant' (uncorrelated) sites will thus always be very inefficient, and it would be highly advantageous to adapt bond dimensions in a site-specific way that efficiently represents the strongly inhomogeneous entanglement properties of the system-environment chains.

Haegeman et al. $\stackrel{26}{ }$ proposed a two-site variant of TDVP (2TDVP) which addressed this issue by expanding the MPS manifold upon which one optimises to include two-site variations of the MPS. This leads to a modified algorithm wherein pairs of neighbouring MPS sites are updated together and subsequently split up via an SVD decomposition. The truncation of singular values which is necessary to prevent bond dimensions exploding, leads to, on the one hand, MPS bond dimensions which can grow to adapt to the entanglement induced by the time evolution, but on the other, a breaking of the appealing geometric properties of the original TDVP method. The error caused by this truncation can lead to a slower convergence for certain observables than would be obtained with the original, one-site form of TDVP (referred to henceforth as 1TDVP) 23134. However, in the context of OQS which is the focus of this paper, there exists a stronger reason why 2TDVP is disadvantageous: the poor scaling in the local Hilbert space dimension of the MPS sites $d$. Since environmental excitations are normally bosonic in nature, $d$ becomes a convergence parameter, and large values $(d \sim 10-100)$ can often be necessary due to strong interactions or finitetemperature effects 8119120149 .

That there is a need for an MPS time evolution algorithm that retains the desirable properties of the 1TDVP, while allowing bond dimensions to change in response to emerging entanglement, is widely agreed upon in the literature and there have recently been a number of papers which make progress in this direction $10|15 / 59| 62$. Notably, Yang and White ${ }^{62}$ have proposed a general, bond-adaptive 1TDVP variant, drawing on ideas related to subspace expansions for one-site $\mathrm{DMRG}^{29}$, wherein the MPS is enriched at each step with the help of global Krylov vectors, which are constructed by several applications of the Hamiltonian's MPO to the MPS. While conceptually different from the unoptimized expansion approach that we shall develop in this article, the global subspace expansion TDVP (GSE-TDVP) of Ref.62] allows bond dimensions to grow during time-evolution with numerical costs and speeds that are intermediate between standard, fixed- $D$ 1TDVP and 2TDVP. Moreover, GSE-TDVP is also able to handle certain classes of long-range problems for which both 1TDVP and 2TDVP will fail. However, this method employs full SVDs to extend the MPS basis, thus incurring a cubic scaling in the local Hilbert dimensions of the sites ${ }^{62}$. Again, given our focus on simulating open quantum systems with strongly excited bosonic environments, it would be advantageous to avoid SVDs and maintain the linear $d$-scaling of standard 1TDVP, if possible.

In this paper we show that this is indeed possible and we present an alternative approach to subspace expansion in 1TDVP which we have developed specifically with OQS problems in mind. Our approach, which we call Adaptive 1TDVP (A1TDVP), is simple and cheap, and exploits state enrichment without the need for singular value decompositions. Moreover, the accuracy of the algorithm is controlled by a single parameter, avoiding the need for fine tuning. By contrast, the more generally applicable GSE-TDVP requires at least two such tuning/error parameters ${ }^{62}$. Essentially, and as will be described, the simplicity of our method stems from the fact that the states used for subspace expansion are unoptimized, which we find nevertheless leads to a bond dimension growth comparable to that generated by 2TDVP. The key innovation is the convergence criterion that is used to chose new values for the bond dimensions.

The structure of this paper is as follows: in Sec. II we will introduce the concepts of MPS and subspace expansion and then, in Sec. III and IV we will explain the A1TDVP method; finally, in Sec. $\mathrm{V}$ we present numeri- 
cal results which show that A1TDVP gives an order of magnitude speed-up over both one- and two-site TDVP.

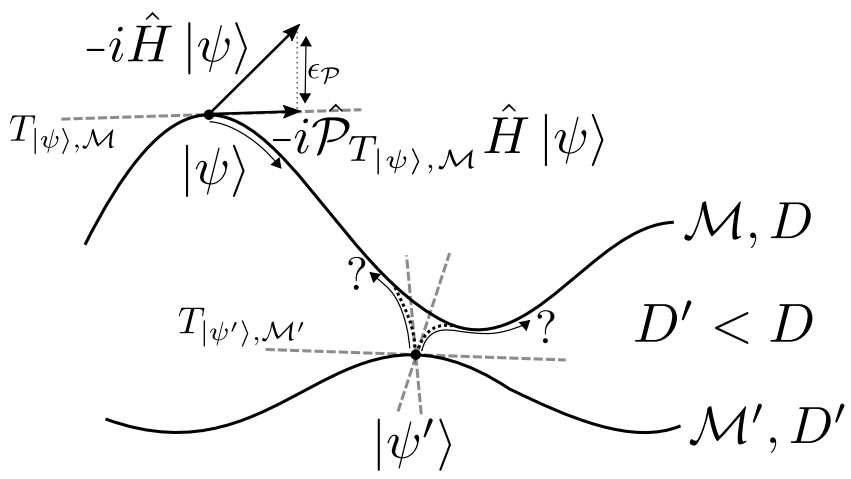

Figure 1. A cartoon illustrating the TDVP. The wavefunction $|\psi\rangle$, with bond dimension $D$, is a point on the fullranked fixed bond dimension MPS manifold $\mathcal{M}$. Evolution under the Hamiltonian $\hat{H}$ would in general take $|\psi\rangle$ out of this manifold and into one of a higher bond dimension. Applying the projector onto the tangent space $T_{|\psi\rangle, \mathcal{M}}$ constrains the evolution to $\mathcal{M}$. The wavefunction $\left|\psi^{\prime}\right\rangle$ is a point on the manifold $\mathcal{M}^{\prime}$ with smaller bond dimension $D^{\prime}$. One may extend the manifold $\mathcal{M}$ to include rank-deficient MPS in order to incorporate the point $\left|\psi^{\prime}\right\rangle$. However, $\left|\psi^{\prime}\right\rangle$ would correspond to a singular point on this manifold where there is no unique tangent space. If one applied the TDVP to $\left|\psi^{\prime}\right\rangle$ in the manifold $\mathcal{M}$, the evolution would be indeterminate. In practice however, this indeterminacy is resolved by the gauge of $\left|\psi^{\prime}\right\rangle$.

\section{MPS AND SUBSPACE EXPANSION}

We begin by expressing the wave-function $|\Psi(t,\{A\})\rangle$ graphically as an MPS with open boundary conditions made up of the set of tensors $\{A\}$ with local physical Hilbert space dimensions $\{d\}$ and bond dimensions $\{D\}$

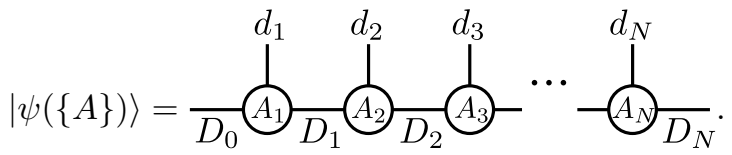

The first and final bond dimension are trivial $\left(D_{0}=\right.$ $\left.D_{N}=1\right)$ such that contracting the entire network, for a choice of physical states, will yield a scalar. Similarly, the Hamiltonian is represented as an MPO

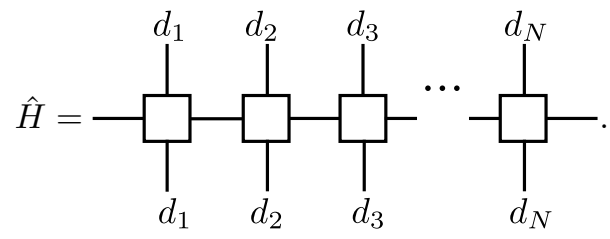

We choose to write our MPS using the convention that a physical leg pointing downwards implies the elements are complex conjugated. Thus the bra is represented as

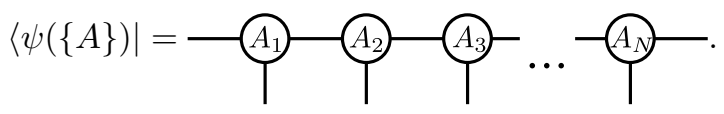

One of the key properties of the MPS representation is its gauge freedom which allows different sets of tensors to represent the same physical state. For example, the transformation $A_{i} \rightarrow A_{i} X, A_{i+1} \rightarrow X^{-1} A_{i+1}$ for any non-singular matrix $X$, leaves the state unchanged. In particular, by performing QR factorizations, one can put the MPS into the so called canonical forms which are the basis of many MPS based algorithms.

The QR factorization takes an $m \times n$ rectangular matrix $A$, where $m \geq n$, and decomposes it into an $m \times m$ unitary matrix $Q$ and an $m \times n$ upper triangular matrix $R$. Since $R$ is upper triangular, its bottom $m-n$ rows are all zero, leading to the following block structure

$$
A=Q R=\left(\begin{array}{ll}
Q_{1} & Q_{2}
\end{array}\right)\left(\begin{array}{c}
R_{1} \\
0
\end{array}\right)=Q_{1} R_{1} .
$$

The matrix $Q_{2}$ consists of $m-n$ orthonormal columns which are orthogonal to the $n$ columns of $Q_{1}$. Since on multiplying together the factors $Q$ and $R$ the block $Q_{2}$ will simply meet the zero rows of $R$, this block is often discarded and the factorization is taken as $Q_{1} R_{1}$. This is known as the thin or reduced QR factorization whereas taking $Q R$ is known as full QR. It should be noted that while $Q_{1}$ is unique (provided that $A$ is full ranked), $Q_{2}$ is not.

Applying this factorization to the tensors in our MPS allows us to decompose $A$ as

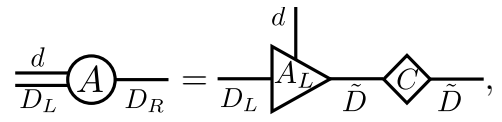

where $A_{\mathrm{L}}$ has the property

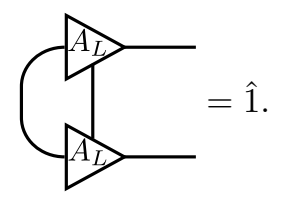

We have written the right bond dimension of $A_{\mathrm{L}}$ above as $\tilde{D}$ to include the possibility of including the one or more of the columns of $Q_{2}$. We may take $\tilde{D}$ to be any value between $D_{\mathrm{R}}$ and $d D_{\mathrm{L}}$ inclusive. Taking $\tilde{D}=D_{\mathrm{R}}$ would correspond to thin $\mathrm{QR}$, while taking $\tilde{D}=d D_{\mathrm{L}}$ would correspond to a full QR. 
It is useful to consider the matrix $A$ as a basis transformation whose job is to take the combined Hilbert space of the $D_{\mathrm{L}}$ states from its left plus the $d$ local states and to find the $D_{\mathrm{R}}$ most relevant states (where often $D_{\mathrm{R}} \ll d D_{\mathrm{L}}$ ) which it then outputs to the next tensor on the chain. This is how the MPS is able to describe many-body quantum states using a computationally viable number of parameters. Including the extra states of $Q_{2}$ can be considered as completing the truncated basis of $D_{\mathrm{R}}$ states such that $A$ outputs either a full basis for the $d D_{\mathrm{L}}$ dimensional Hilbert space, or a less severely truncated one. This is what we mean by subspace expansion. Of course, for a given MPS, including the extra $Q_{2}$ states will make no difference since the next tensor along the chain will still only except $D_{\mathrm{R}}$ states from its left. However, this notion of sub-space expansion will be in important in Sec. III when we introduce the A1TDVP method.

We can equally take the mirror image of Eq. (6) and decompose $A$ as

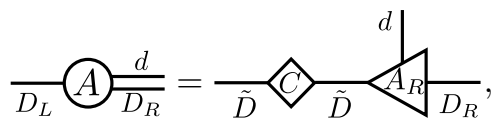

where now

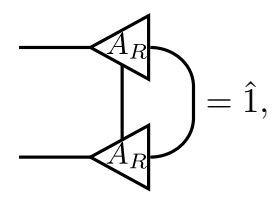

and $D_{\mathrm{L}} \leq \tilde{D} \leq d D_{\mathrm{R}}$.

By always taking thin QRs we can put the MPS into canonical form by iteratively applying Eq. (8) from the right and Eq. 6) from the left and contracting $C$ into the neighboring site

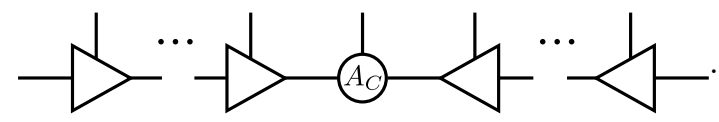

In doing so one will always be left with one site that is not of the form $A_{\mathrm{L}}$ or $A_{\mathrm{R}}$. This site will be known as the orthogonality center and will be denoted $A_{\mathrm{C}}$. The orthogonality center may be placed on any site of the MPS. If $A_{\mathrm{C}}$ is on site $1(N)$ the MPS is said to be in right(left)-canonical form, while its being on any other site is known as mixed-canonical form.

One may also gauge the MPS such that $C$ lies between two sites

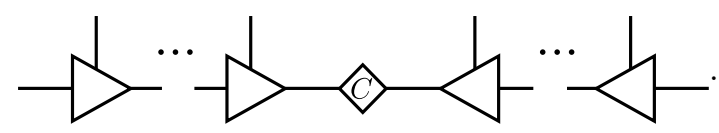

\section{FROM 1TDVP TO A1TDVP}

The key insight behind 1TDVP is that the tangent space projector appearing in Eq. (1) can be exactly decomposed into a sum of terms $i$ that each project out all but the degrees of freedom associated with either a given physical site $i$ or the bond between sites $i, i+1^{37}$

$$
\hat{\mathcal{P}}_{T_{|\psi\rangle, \mathcal{M}}}(\{\tilde{D}\})=\sum_{i=1}^{N} \hat{P}_{A_{\mathrm{C}}}^{(i)}\left(\tilde{D}_{i-1}, \tilde{D}_{i}\right)-\sum_{i=1}^{N-1} \hat{P}_{\mathrm{C}}^{(i)}\left(\tilde{D}_{i}\right),
$$

with



and

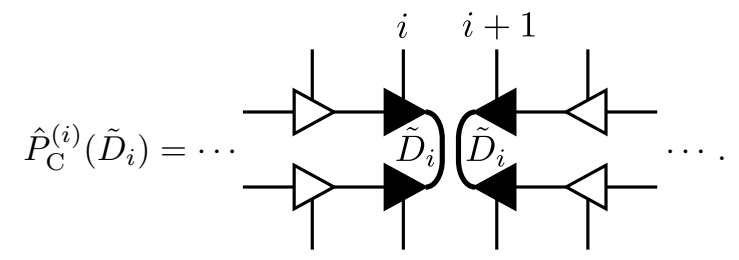

In conventional 1TDVP the projectors are constructed from the MPS using thin QR decompositions, however, in the above we have introduced a dependence on the bond dimension by expanding certain sites in the manner described in Sec. III We indicate an MPS site that has been expanded using a filled black tensor. In this way, the application of $\hat{\mathcal{P}}_{T_{|\psi\rangle, \mathcal{M}}}(\{\tilde{D}\})$ will result in a projection onto the manifold of MPS with the chosen bond dimensions $\{\tilde{D}\}$. Of course, choosing $\{\tilde{D}\}=\{D\}$ will result in the original projector used in 1TDVP.

This procedure is equivalent to constructing the projectors from a rank-deficient MPS $\left|\psi^{\prime}\right\rangle$, which would correspond to a singular point on $\mathcal{M}$ (see Fig. 1 ${ }^{25}$. At such points the tangent space is not well defined by the wavefunction alone but will also depend on the gauge of $\left|\psi^{\prime}\right\rangle$. In the present case this means that the projector of Eq. $\sqrt{12}$, and thus the projection error, will have a dependence on the states $Q_{2}$ that are used to expand the subspaces. The $Q_{2}$ matrices which are output by the $\mathrm{QR}$ routine should be in no way optimized to minimise the projection error. We find however (see Sec. V) that it is sufficient to consider only these unoptimised states to produce an algorithm that significantly outperforms 2TDVP. 
The reason for splitting the projector in this manner is that, on substituting Eq. 12 into Eq. 11 each term may be integrated exactly 37 . For example, by gauging the MPS as in Eq. 10 with $A_{\mathrm{C}}$ on site $i$ the operator $\hat{P}_{A_{\mathrm{C}}}^{(i)} \hat{H}$ affects only site $A_{\mathrm{C}}$ and so may be written as an effective Hamiltonian $H_{\mathrm{eff}}^{i}$ which acts only on this site
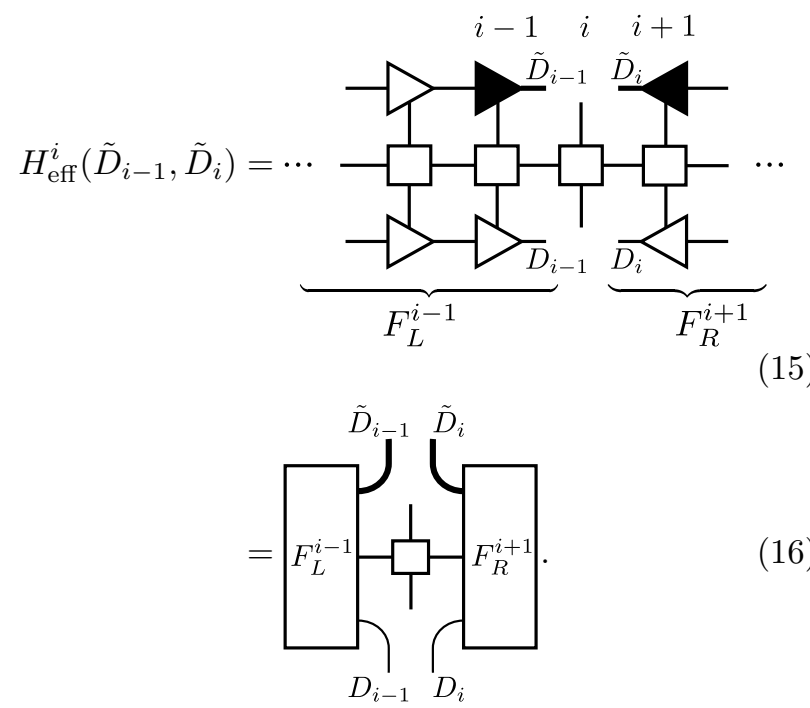

Then by making all other sites time-independent we can write the exact evolution of $A_{\mathrm{C}}$ as

$$
A_{\mathrm{C}}^{i}(t)=\exp \left[-i H_{\mathrm{eff}}^{i} t\right] A_{\mathrm{C}}^{i}(0) .
$$

Similarly by writing the MPS as in Eq. 11 with $C$ between sites $i$ and $i+1$ and making only $C$ timedependent we have

$$
C^{i}(t)=\exp \left[+i K_{\mathrm{eff}}^{i} t\right] C^{i}(0)
$$

with the effective Hamiltonian

$$
K_{\mathrm{eff}}^{i}\left(\tilde{D}_{i}\right)=F_{L}^{i} F_{D_{i}}^{F_{D_{i}}}
$$

With these solutions the entire MPS can be evolved using a Lie-Trotter splitting $\sqrt{16|36| 58}$ by sweeping from left to right along the chain and evolving each $A_{\mathrm{C}}$ and $C$ by a time step $\Delta t$. If this left to right sweep is composed with a reverse sweep from right to left then this procedure constitutes a second-order integrator with error $O\left(\Delta t^{3}\right)$.

With the sub-space expansions employed in Eq. 12 the effective Hamiltonians become capable of increasing the bond dimensions, whereas in normal 1TDVP they would leave them unchanged. For example, $H_{\text {eff }}^{i}\left(\tilde{D}_{i-1}, \tilde{D}_{i}\right)$ takes an MPS site tensor $A_{\mathrm{C}}$ with right and left bond dimensions $D_{i-1}$ and $D_{i}$ respectively and outputs a tensor with bond dimensions $\tilde{D}_{i-1}$ and $\tilde{D}_{i}$. In the next section we will explain how these new values for the bond dimensions are chosen in A1TDVP.

\section{PROJECTION ERROR}

Ideally, bond dimensions would be chosen so as to guarantee that the projection error $\epsilon_{\mathcal{P}}$ does not exceed a certain acceptable value: $\epsilon_{\mathcal{P}} \leq \epsilon_{\mathcal{P}}^{\prime}$. The projection error arises from the fact that the true time evolution vector $\hat{H}|\psi\rangle$ differs from the projected one $\hat{\mathcal{P}}_{T_{|\psi\rangle, \mathcal{M}}} \hat{H}|\psi\rangle$ by a residue $|\phi\rangle$

$$
|\phi\rangle:=\hat{H}|\psi\rangle-\hat{\mathcal{P}}_{T_{|\psi\rangle, \mathcal{M}}}|\psi\rangle .
$$

We can thus define the projection error as the norm of this residue

$$
\begin{aligned}
\epsilon_{\mathcal{P}}(\{\tilde{D}\}) & :=\langle\phi \mid \phi\rangle \\
& =\left\langle\psi\left|\hat{H}^{2}\right| \psi\right\rangle-\left\langle\psi\left|\hat{H} \hat{\mathcal{P}}_{T_{\left|\psi^{\prime}\right\rangle, \mathcal{M}}}(\{\tilde{D}\}) \hat{H}\right| \psi\right\rangle,
\end{aligned}
$$

where we have inserted the bond dimension dependent projectors to obtain the projection error as a function of $\{\tilde{D}\}$. A full evaluation of $\epsilon_{\mathcal{P}}$ will be often be prohibitively expensive ${ }^{29}$ and would always have a greater computational complexity than a 1TDVP sweep. However, we see that in Eq. 22 only the second term has a dependence on the new bond dimensions $\{\tilde{D}\}$; thus, the effect that increasing the bond dimensions has on the projection error should be entirely contained within this term. Further inserting the decomposition of the projection (Eq. (12) ) into Eq. (22), we see that the effect of changing only one bond dimension $\tilde{D}_{i}$ while keeping all the rest constant is described by the function

$$
\begin{aligned}
f_{i}\left(\tilde{D}_{i}\right):=\| \hat{H}_{\mathrm{eff}}^{i}\left(D_{i-1},\right. & \left.\tilde{D}_{i}\right) A_{\mathrm{C}}^{i}\left\|^{2}-\right\| \hat{K}_{\mathrm{eff}}^{i}\left(\tilde{D}_{i}\right) C^{i} \|^{2} \\
& +\left\|\hat{H}_{\mathrm{eff}}^{i+1}\left(\tilde{D}_{i}, D_{i+1}\right) A_{\mathrm{C}}^{i+1}\right\|^{2}
\end{aligned}
$$

We find that choosing bond dimensions to satisfy the requirement

$$
\frac{1}{f_{i}\left(\tilde{D}_{i}\right)} \frac{\partial f_{i}}{\partial \tilde{D}_{i}} \leq p
$$

where $p$ defines a precision, works well in economising resources whilst providing fast convergence. The presence of the factor of $f_{i}\left(\tilde{D}_{i}\right)$ in the denominator makes the convergence criterion independent of the scale of $f$. 
This is important because the magnitude of $f_{i}\left(\tilde{D}_{i}\right)$ has no meaning on its own as it only represents half of the expression for the projection error (Eq. (21)).

In only considering the dependence of the projection error on each bond dimension individually, we ignore any possible co-dependence of the bond dimensions, which may arise from the $\hat{H}_{\text {eff }}^{i}$ terms in Eq. (23). New values $D_{i}^{\prime}$ for each bond dimension can be determined by evaluating the left hand side of Eq. (24) at incrementally increasing values of $\tilde{D}_{i}$, for each site $i$, until the inequality is satisfied.

The procedure of updating the bond dimensions with this measure is inexpensive and costs as follows. First the tensors $F_{L}^{i}$ defined in Eq. 15 for $i \in[1, N-1]$ must be computed, requiring a left to right $\mathrm{QR}$ sweep of the MPS. The tensors $F_{R}^{i}$ will already be available from the previous right to left sweep of 1TDVP. The overhead of this additional QR sweep may be mitigated by using the $A_{\mathrm{CS}}$ produced as a shortcut to computing observables along the chain for that timestep. Following this, the quantities $f_{i}\left(\tilde{D}_{i}\right)$ may be computed.

It is clear that this bond-update step will take only a small fraction (typically $<10 \%$ ) of the time required for a 1TDVP sweep. In 1TDVP by far the most expensive operation is the application of the exponentiated effective Hamiltonians which are the formal solutions of the 1TDVP equations of motion (cf. Eq. (17) and (18)). These are carried out using the Krylov method ${ }^{28}$, which involves many repeated applications of $\hat{H}_{\text {eff }}^{i}$ and $\hat{K}_{\text {eff }}^{i}$ respectively. In the adaptive bond update step these operations are replaced by one calculation of $\hat{H}_{\text {eff }}^{i} A_{\mathrm{C}}^{i}$ and $\hat{K}_{\text {eff }}^{i} C^{i}$ which each require only a single application of $\hat{H}_{\text {eff }}^{i}$ and $\hat{K}_{\text {eff }}^{i}$, respectively.

Using the convergence measure (24) avoids the need to ever deal with the fully expanded subspaces of size $d D$ as in 2TDVP, and indeed one can avoid computing tensors with dimensions greater than what will finally be taken as the new bond dimensions $\left\{D^{\prime}\right\}$. Thus, and herein lies the main virtue of our method, the complexity of A1TDVP is limited to that of 1TDVP. Furthermore, since individual bond dimensions are chosen independently, they may be computed in parallel, leading to an up to $(N-1)$-fold speed-up.

\section{NUMERICAL VERIFICATION}

We now demonstrate our method through simulations of a two-level system (TLS) strongly coupled to two continuous bosonic baths at different temperatures (Fig. 3). This class of model has wide applications, including excitation transfer, quantum thermodynamics, clocks, and non-equilibrium steady states/5/111/17/18|24]38/52/63. Importantly for us, this entropy generating system shows strong and inhomogeneously growing entanglement (bond dimensions) that A1TDVP can track from a simple, low bond dimension initial state. The microscopic Hamiltonian has the standard open system form $H=H_{S}+H_{B}+H_{I}$, where $H_{S}, H_{B} \& H_{I}$ are the system, environment and interaction terms, respectively. The TLS has $H_{S}=\frac{\omega_{0}}{2} \sigma_{z}$, and $H_{B}=\sum_{i, k} \omega_{k} \hat{a}_{i k}^{\dagger} \hat{a}_{i k}$, where $i=\mathrm{h}, \mathrm{c}$ indicates the hotter (h) or colder (c) reservoir, respectively. The interaction term induces TLS transitions according to $H_{I}=\frac{\sigma_{x}}{2} \otimes \sum_{i, k}\left(g_{k}^{*} \hat{a}_{i k}+g_{k} \hat{a}_{i k}^{\dagger}\right)$. To explore effects from temperature differences, we take both baths to have the same Ohmic spectral function $J(\omega) \equiv \sum_{k}\left|g_{k}\right|^{2} \delta\left(\omega-\omega_{k}\right)=2 \alpha \omega \theta\left(\omega_{\mathrm{c}}-\omega\right)$. The cut-off frequency of the bath $\omega_{c}$ is taken as the unit of energy and set to unity in the following.

The initial condition is given by the product density matrix $\rho(0)=\left|\uparrow_{z}\right\rangle\left\langle\uparrow_{z}\right| \otimes \rho_{\mathrm{h}} \otimes \rho_{\mathrm{c}}$, where $\rho_{i}$ is the thermal density matrix for the $i$ th (uncoupled) environment at inverse temperature $\beta_{i}(\beta=1 / T)$. Such a mixed-state initial condition is, prima facie, a major problem: each single wave function simulation is costly, so sampling over thermal initial conditions would be utterly intractable. However, here we exploit the T-TEDOPA chain mapping $12|14| 56$, which maps the system-bath problem into a $1 D$ nearest-neighbour problem where the effects of temperature are encoded only in the chains' Hamiltonians (see Fig. 3), rather than the mixedness of the initial state. Finite temperature effects can then be extracted from the evolution of the uncorrelated pure initial state $|\psi\rangle=\otimes\left|\uparrow_{z}\right\rangle \otimes|0\rangle_{\mathrm{h}} \otimes|0\rangle_{\mathrm{c}}$, where $|0\rangle_{i}$ is the vacuum state of bath $i$.

We ran A1TDVP on this model for several values of the precision $p$ and compared the resulting dynamics and bond dimensions against those obtained using 2TDVP, also for different precisions. We also calculated the dynamics with 1TDVP at a very large bond dimension $\left(D_{\max }=120\right)$ to provide a highly converged, benchmark result for evaluating the convergence of the bond adaptive simulations. As is common in bond adaptive methods, to avoid exploding memory usage, we set an upper bound $D_{\text {lim }}$ for the bond dimensions. We chose $D_{\lim }=120$ for both the A1TDVP and 2TDVP simulations.

Figure 2 shows the observable $\left\langle\sigma_{z}\right\rangle$ obtained using A1TDVP at four different precisions. It was found that a local Hilbert space dimension of $d=15$ for all chain oscillators present in the simulation, was sufficient to give well converged results. The chains for both baths were truncated so as to contain 40 sites each. The structure of the MPS is as shown above Fig. 3 with the TLS on site 41 , between the two baths. We find that a precision of $p=10^{-4}$ gives well converged results up to a time of $\omega_{\mathrm{c}} t=25$.

In Fig. 3 we plot the MPS bond dimensions at 


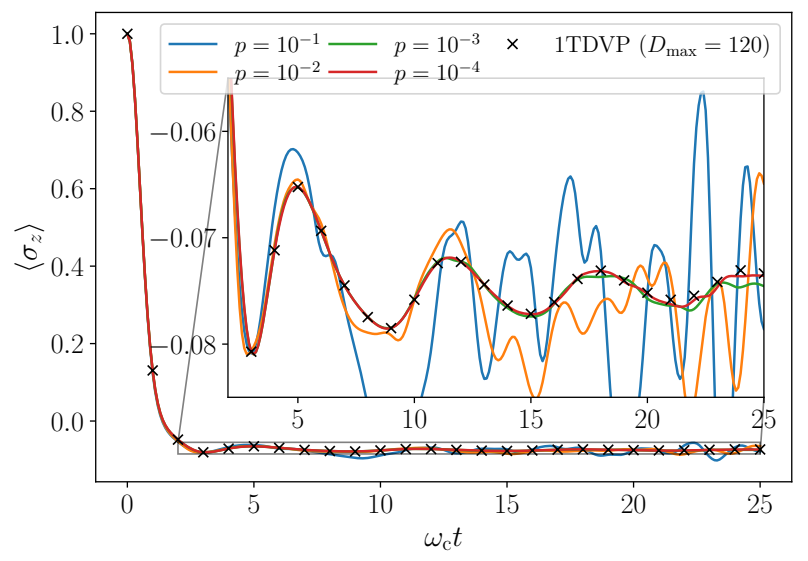

Figure 2. $\left\langle\sigma_{z}(t)\right\rangle$ for $\omega_{0}=0.2 \omega_{\mathrm{c}}, \beta_{\mathrm{c}}=100 / \omega_{\mathrm{c}}, \beta_{\mathrm{h}}=1 / \omega_{\mathrm{c}}$, $\alpha=0.2$ and 15 Fock states per oscillator. Different precisions $p$ with $D_{\lim }=120$ are compared against standard 1TDVP with $D_{\max }=120$ (crosses). Inset shows an expanded view of $\left\langle\sigma_{z}(t)\right\rangle$ demonstrating the convergence of our adaptive TDVP.

three snapshots in time for the different precisions of A1TDVP. We observe that the distribution of resources is skewed towards the hotter environment, both in terms of magnitude and propagation speed along the chain. Also shown in Fig. 3 are the bond dimensions given by 2TDVP at the precision of $p=10^{-4}$ which we found to give a similar quality of result as A1TDVP for $p=10^{-439}$. Comparing the bond dimensions for these two cases, we see that they have a similar distribution and amplitude, suggesting that it is sufficient to use unoptimised states for subspace expansion.

All simulations were performed on nodes consisting of two 12-core Intel Xeon Haswell (E5-2670v3) processors. The total run times for the three methods are given in Tab. I. The speed-up achieved by A1TDVP is quite striking, being almost 10 times faster than 1TDVP and 30 times faster than 2TDVP. Of course, the comparison between 1TDVP and A1TDVP would be less favourable to A1TDVP had we chosen the chains to be shorter; by the end of the simulation at $\omega_{\mathrm{c}} t=25$ the perturbation has travelled about 30 sites into the hot bath's chain and about 20 sites into the cold bath's chain, and so we could have truncated them more severely without affecting the results. However, such optimisations are only possible with hindsight, as we do not know how the light-cone will spread before we run the simulation ${ }^{40}$. The efficiency of A1TDVP on the other hand is relatively insensitive to the chosen chain length, since it takes very little time to update MPS sites for which the bond dimensions are trivial. Finally, we note that this is also true when long-range interactions are present in
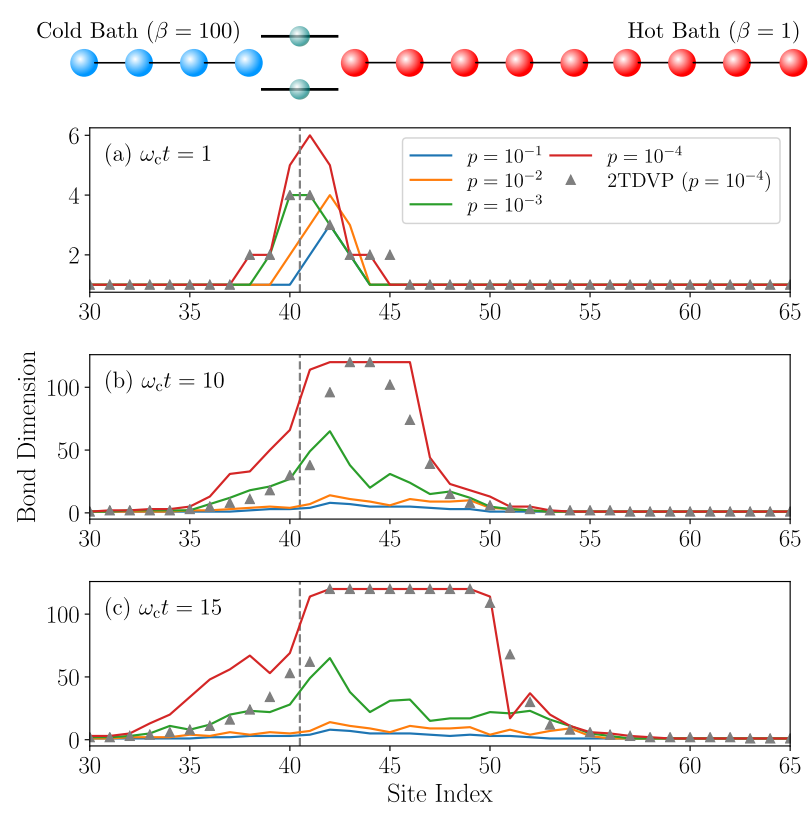

Figure 3. MPS bond dimensions for snapshots at times $\omega_{\mathrm{c}} t=$ 1 (panel 'a'), $\omega_{\mathrm{c}} t=10$ (panel 'b'), and $\omega_{\mathrm{c}} t=15$ (panel 'c') during A1TDVP simulations at various precisions and the converged 2TDVP simulation at precision $p=10^{-4}$. The grey dashed line, plotted at $x$-position 40.5 , indicates the position of the TLS; all bond dimensions to the right of this line pertain to the hot bath sites, and all bond dimensions to its left pertain to the cold bath sites.

the system-chain Hamiltonian. These interactions arise when the fluctuations of the environment act on the system states in a correlated fashion, such as those arsing as a result of the spatial correlations, i.e. separated system sites interacting with extended plane-wave excitations of their common environment ${ }^{33}$. Indeed, accounting for correlations in environmental fluctuations has recently been shown to be essential in extracting realistic optical spectra from $a b$ inito models of molecular excited states $^{21}$, and A1TDVP has been demonstrated to provide a highly efficient technique for handling these more complex system-environment Hamiltonians ${ }^{41}$.

\section{CONCLUSION}

In this paper we have presented a bond adaptive variant of 1TDVP, which we have demonstrated to be highly advantageous for the OQS problem. The features of the OQS problem that make it particularly well suited to A1TDVP are its light-cone structure and the fact that its favourable scaling in the local physical dimension $d$ is advantageous for typical bosonic environ- 


\begin{tabular}{l|c} 
Method & Run Time (mins) \\
\hline \hline $1 \operatorname{TDVP}\left(D_{\max }=120\right)$ & 338 \\
$2 \operatorname{TDVP}\left(p=10^{-4}, D_{\text {lim }}=120\right)$ & 1054 \\
$\operatorname{A} 1 \operatorname{TDVP}\left(p=10^{-4}, D_{\text {lim }}=120\right)$ & $35^{\mathrm{a}}$
\end{tabular}

a This time does not include the time required for the bond update step, which we were not able to obtain. However, since the bond update step is expected to take a small fraction of the time required for TDVP, this will not affect our conclusions.

Table I. Run times for converged simulations under the three methods considered.

ments - especially so when thermal chain mappings are employed $19 \mid 20$.
This idea may be straight forwardly extended to tree-MPS which will allow the simulation of systems with complex multi-environment interactions, whereupon, the advantages demonstrated here, especially the parallelizable steps, will become even more important and could be further combined with the 'entanglement renormalisation techniques' introduced in Ref. 51 for $a b$ initio open quantum dynamics. A basic demonstration of this has recently been given for the three-environment tree network that describes the photophysics of the dye Methyl-Blue ${ }^{21}$. Beyond our present focus on open quantum systems, we also hope that our approach could be readily applied to a range of other problems in condensed or atomic quantum matter and - as suggested by our heat-flow example - the microscopic analysis of more complex nanodevices operating in the regime of quantum thermodynamics.
1 Antonio Acín, Immanuel Bloch, Harry Buhrman, Tommaso Calarco, Christopher Eichler, Jens Eisert, Daniel Esteve, Nicolas Gisin, Steffen J Glaser, Fedor Jelezko, et al. The quantum technologies roadmap: a european community view. New Journal of Physics, 20(8):080201, 2018.

2 Robert Alicki and Ronnie Kosloff. Introduction to quantum thermodynamics: History and prospects. In Thermodynamics in the Quantum Regime, pages 1-33. Springer, 2018.

${ }^{3}$ Yuto Ashida. Quantum Many-Body Physics in Open Systems: Measurement and Strong Correlations. Springer Nature, 2020.

${ }^{4}$ Daniel Bauernfeind and Markus Aichhorn. Time Dependent Variational Principle for Tree Tensor Networks. SciPost Physics, 8(2):024, February 2020. ISSN 25424653. doi:10.21468/SciPostPhys.8.2.024 URL http: //arxiv.org/abs/1908.03090 arXiv: 1908.03090.

'Giuliano Benenti, Giulio Casati, Keiji Saito, and Robert S. Whitney. Fundamental aspects of steadystate conversion of heat to work at the nanoscale. Physics Reports, 694:1-124, June 2017. ISSN 03701573. doi:10.1016/j.physrep.2017.05.008 URL http://www.sciencedirect.com/science/article/ pii/S0370157317301540.

6 Jean-Luc Brédas, Edward H Sargent, and Gregory D Scholes. Photovoltaic concepts inspired by coherence effects in photosynthetic systems. Nature materials, 16(1):35-44, 2017.

7 Heinz-Peter Breuer, Francesco Petruccione, et al. The theory of open quantum systems. Oxford University Press on Demand, 2002.

${ }^{8}$ Christoph Brockt, Florian Dorfner, Lev Vidmar, Fabian Heidrich-Meisner, and Eric Jeckelmann. Matrix-productstate method with a dynamical local basis optimization for bosonic systems out of equilibrium. Physical Review
$B, 92(24): 241106,2015$.

9 Benedikt Bruognolo, Andreas Weichselbaum, Cheng Guo, Jan von Delft, Imke Schneider, and Matthias Vojta. Two-bath spin-boson model: Phase diagram and critical properties. Physical Review B, 90(24):245130, December 2014. ISSN 1098-0121, 1550-235X. doi: 10.1103/PhysRevB.90.245130. URL https://link.aps . org/doi/10.1103/PhysRevB.90.245130.

I0 Gianluca Ceruti, Jonas Kusch, and Christian Lubich. A rank-adaptive robust integrator for dynamical lowrank approximation. arXiv:2104.05247 [cs, math], April 2021. URL http://arxiv.org/abs/2104.05247. arXiv: 2104.05247.

11 Tianqi Chen, Vinitha Balachandran, Chu Guo, and Dario Poletti. Steady state quantum transport through an anharmonic oscillator strongly coupled to two heat reservoirs. arXiv:2004.05017 [cond-mat], April 2020. URL http://arxiv.org/abs/2004.05017, arXiv: 2004.05017.

12 Alex W. Chin, Angel Rivas, Susana F. Huelga, and Martin B. Plenio. Exact mapping between system-reservoir quantum models and semi-infinite discrete chains using orthogonal polynomials. Journal of Mathematical Physics, 51(9):092109, September 2010. ISSN 0022-2488, 1089-7658. doi:10.1063/1.3490188 URL http://arxiv . org/abs/1006.4507 arXiv: 1006.4507.

13 AW Chin, J Prior, R Rosenbach, F Caycedo-Soler, Susana F Huelga, and Martin B Plenio. The role of nonequilibrium vibrational structures in electronic coherence and recoherence in pigment-protein complexes. Nature Physics, 9(2):113-118, 2013.

14 Ines de Vega, Ulrich Schollwöck, and F. Alexander Wolf. How to discretize a quantum bath for realtime evolution. Physical Review B, 92(15):155126, October 2015. ISSN 1098-0121, 1550-235X. doi: 10.1103/PhysRevB.92.155126 URL http://arxiv.org/ abs/1507.07468 arXiv: 1507.07468. 
15 Alec Dektor, Abram Rodgers, and Daniele Venturi. Rankadaptive tensor methods for high-dimensional nonlinear PDEs. arXiv:2012.05962 [physics], April 2021. URL http://arxiv.org/abs/2012.05962 arXiv: 2012.05962.

16 Stéphane Descombes and Mechthild Thalhammer. The Lie-Trotter splitting for nonlinear evolutionary problems with critical parameters: a compact local error representation and application to nonlinear Schrödinger equations in the semiclassical regime. IMA Journal of Numerical Analysis, 33(2):722-745, April 2013. ISSN 0272-4979. doi: 10.1093/imanum/drs021. URL https://academic.oup. com/imajna/article/33/2/722/653345. Publisher: Oxford Academic.

17 Abhishek Dhar. Heat transport in low-dimensional systems. Advances in Physics, 57(5):457537, September 2008. ISSN 0001-8732, 14606976. doi:10.1080/00018730802538522. URL http://www.tandfonline.com/doi/abs/10.1080/ 00018730802538522

18 Yonatan Dubi and Massimiliano Di Ventra. Colloquium : Heat flow and thermoelectricity in atomic and molecular junctions. Reviews of Modern Physics, 83(1):131155, March 2011. ISSN 0034-6861, 1539-0756. doi: 10.1103/RevModPhys.83.131. URL https://link.aps . org/doi/10.1103/RevModPhys.83.131.

19 Angus J Dunnett and Alex W Chin. Matrix product state simulations of non-equilibrium steady states and transient heat flows in the two-bath spin-boson model at finite temperatures. Entropy, 23(1):77, 2021.

20 Angus J. Dunnett and Alex W. Chin. Simulating quantum vibronic dynamics at finite temperatures with many body wave functions at 0 k. Frontiers in Chemistry, 8:1195, 2021. ISSN 2296-2646. doi:10.3389/fchem.2020.600731. URL https://www.frontiersin.org/article/10.3389/ fchem.2020.600731

21 Angus J Dunnett, Duncan Gowland, Christine M Isborn, Alex W Chin, and Tim J Zuehlsdorff. Influence of nonadiabatic effects on linear absorption spectra in the condensed phase: Methylene blue. The Journal of Chemical Physics, 155(14):144112, 2021.

22 C. Gonzalez-Ballestero, Florian A. Y. N. Schröder, and Alex W. Chin. Uncovering nonperturbative dynamics of the biased sub-ohmic spin-boson model with variational matrix product states. Phys. Rev. B, 96:115427, Sep 2017. doi:10.1103/PhysRevB.96.115427 URL https://link. aps.org/doi/10.1103/PhysRevB.96.115427.

23 Shimpei Goto and Ippei Danshita. Performance of the time-dependent variational principle for matrix product states in long-time evolution of a pure state. Physical Review B, 99(5):054307, February 2019. ISSN 2469-9950, 2469-9969. doi:10.1103/PhysRevB.99.054307. URL http: //arxiv.org/abs/1809.01400, arXiv: 1809.01400.

${ }^{24}$ Cheng Guo, Andreas Weichselbaum, Jan von Delft, and Matthias Vojta. Critical and Strong-Coupling Phases in One- and Two-Bath Spin-Boson Models. Physical Review Letters, 108(16):160401, April 2012. ISSN 0031-9007, 1079-7114. doi:10.1103/PhysRevLett.108.160401. URL https://link.aps.org/doi/10.1103/PhysRevLett.
108.160401

25 Jutho Haegeman, J. Ignacio Cirac, Tobias J. Osborne, Iztok Pižorn, Henri Verschelde, and Frank Verstraete. Time-Dependent Variational Principle for Quantum Lattices. Physical Review Letters, 107(7):070601, August 2011. ISSN 0031-9007, 1079-7114. doi: 10.1103/PhysRevLett.107.070601. URL https://link. aps.org/doi/10.1103/PhysRevLett.107.070601

zo Jutho Haegeman, Christian Lubich, Ivan Oseledets, Bart Vandereycken, and Frank Verstraete. Unifying time evolution and optimization with matrix product states. Physical Review B, 94(16):165116, October 2016. ISSN 24699950, 2469-9969. doi:10.1103/PhysRevB.94.165116. URL http://arxiv.org/abs/1408.5056 arXiv: 1408.5056.

27 Markus Hauru, Maarten Van Damme, and Jutho Haegeman. Riemannian optimization of isometric tensor networks. arXiv preprint arXiv:2007.03638, 2020.

28 Marlis Hochbruck and Christian Lubich. On Krylov Subspace Approximations to the Matrix Exponential Operator. SIAM Journal on Numerical Analysis, 34(5):1911-1925, October 1997. ISSN 0036-1429. doi:10.1137/S0036142995280572 URL https://epubs. siam.org/doi/10.1137/S0036142995280572 Publisher: Society for Industrial and Applied Mathematics.

29 C. Hubig, I. P. McCulloch, U. Schollwöck, and F. A. Wolf. Strictly single-site DMRG algorithm with subspace expansion. Physical Review B, 91(15):155115, April 2015. ISSN 1098-0121, 1550-235X. doi: 10.1103/PhysRevB.91.155115. URL https://link.aps . org/doi/10.1103/PhysRevB.91.155115

so C. Hubig, I. P. McCulloch, and U. Schollwöck. Generic Construction of Efficient Matrix Product Operators. Physical Review B, 95(3):035129, January 2017. ISSN 2469-9950, 2469-9969. doi: 10.1103/PhysRevB.95.035129. URL http://arxiv.org/ abs/1611.02498 arXiv: 1611.02498.

31 C. Hubig, J. Haegeman, and U. Schollwöck. Error estimates for extrapolations with matrix-product states. Physical Review B, 97(4):045125, January 2018. ISSN 2469-9950, 2469-9969. doi: 10.1103/PhysRevB.97.045125 URL https://link.aps. org/doi/10.1103/PhysRevB.97.045125

32 Akihito Ishizaki and Graham R Fleming. Unified treatment of quantum coherent and incoherent hopping dynamics in electronic energy transfer: Reduced hierarchy equation approach. The Journal of chemical physics, 130 (23):234111, 2009.

33 Thibaut Lacroix, Angus Dunnett, Dominic Gribben, Brendon W Lovett, and Alex Chin. Unveiling nonmarkovian spacetime signaling in open quantum systems with long-range tensor network dynamics. Physical Review A, 104(5):052204, 2021.

34 Eyal Leviatan, Frank Pollmann, Jens H. Bardarson, David A. Huse, and Ehud Altman. Quantum thermalization dynamics with Matrix-Product States. arXiv:1702.08894 [cond-mat, physics:quant-ph], October 2017. URL http://arxiv.org/abs/1702.08894, arXiv: 1702.08894 .

35 Carsten J Lindner, Fabian B Kugler, Volker Meden, and 
Herbert Schoeller. Renormalization group transport theory for open quantum systems: Charge fluctuations in multilevel quantum dots in and out of equilibrium. Physical Review B, 99(20):205142, 2019.

36 Christian Lubich. From Quantum to Classical Molecular Dynamics: Reduced Models and Numerical Analysis. European Mathematical Society, 2008. ISBN 978-3-03719067-8. Google-Books-ID: 11brUAQLvZoC.

37 Christian Lubich, Ivan Oseledets, and Bart Vandereycken. Time integration of tensor trains. SIAM Journal on Numerical Analysis, 53(2):917-941, January 2015. ISSN 0036-1429, 1095-7170. doi:10.1137/140976546. URL http://arxiv.org/abs/1407.2042 arXiv: 1407.2042.

38 G. J. Milburn. The thermodynamics of clocks. Contemporary Physics, 61(2):69-95, April 2020. ISSN 0010-7514, 1366-5812. doi:10.1080/00107514.2020.1837471. URL http://arxiv.org/abs/2007.02217 arXiv: 2007.02217.

39 Note1. Although we use the same symbol $p$ to denote the precision of both A1TDVP and 2TDVP, this parameter is defined differently for each method.

40 Note2. This is true in general although for the simple case of a nearest-neighbour hopping chain one can quite easily estimate how fast the light-cone will spread.

41 Note3. While these models involve long-range interactions, these interactions only exist between the system 'site' and the sites of the chain. Only nearest-neighbour interactions exist between the environmental chain sites, placing these models in a different class to those studied with GKE-TDVP in Ref!62. This latter class do not - to the best of our knowledge - map onto any of the common open quantum system models, although it would be very interesting to explore what sorts of the environment they do describe.

42 Roman Orus. A Practical Introduction to Tensor Networks: Matrix Product States and Projected Entangled Pair States. Annals of Physics, 349: 117-158, October 2014. ISSN 00034916. doi: 10.1016/j.aop.2014.06.013 URL http://arxiv.org/ abs/1306.2164 arXiv: 1306.2164.

43 S. Oviedo-Casado, J. Prior, A. W. Chin, R. Rosenbach, S. F. Huelga, and M. B. Plenio. Phase-dependent exciton transport and energy harvesting from thermal environments. Phys. Rev. A, 93:020102, Feb 2016. doi: 10.1103/PhysRevA.93.020102, URL https://link.aps. org/doi/10.1103/PhysRevA.93.020102

44 S Oviedo-Casado, J Prior, AW Chin, R Rosenbach, SF Huelga, and MB Plenio. Phase-dependent exciton transport and energy harvesting from thermal environments. Physical Review A, 93(2):020102, 2016.

45 Sebastian Paeckel, Thomas Köhler, Andreas Swoboda, Salvatore R. Manmana, Ulrich Schollwöck, and Claudius Hubig. Time-evolution methods for matrix-product states. Annals of Physics, 411:167998, December 2019. ISSN 00034916. doi:10.1016/j.aop.2019.167998 URL http://arxiv.org/abs/1901.05824 arXiv: 1901.05824.

46 Javier Prior, Alex W Chin, Susana F Huelga, and Martin B Plenio. Efficient simulation of strong systemenvironment interactions. Physical review letters, 105(5): 050404, 2010.
47 Gregory D Scholes, Graham R Fleming, Lin X Chen, Alán Aspuru-Guzik, Andreas Buchleitner, David F Coker, Gregory S Engel, Rienk Van Grondelle, Akihito Ishizaki, David M Jonas, et al. Using coherence to enhance function in chemical and biophysical systems. Nature, 543 (7647):647-656, 2017.

48 Ulrich Schollwöck. The density-matrix renormalization group in the age of matrix product states. Annals of Physics, 326(1):96-192, January 2011. ISSN 00034916. doi:10.1016/j.aop.2010.09.012 URL https://linkinghub.elsevier.com/retrieve/pii/ S0003491610001752

49 Florian A. Y. N. Schröder and Alex W. Chin. Simulating open quantum dynamics with time-dependent variational matrix product states: Towards microscopic correlation of environment dynamics and reduced system evolution. Phys. Rev. B, 93:075105, Feb 2016. doi: 10.1103/PhysRevB.93.075105 URL https://link.aps . org/doi/10.1103/PhysRevB.93.075105.

50 Florian AYN Schröder, David HP Turban, Andrew J Musser, Nicholas DM Hine, and Alex W Chin. Tensor network simulation of multi-environmental open quantum dynamics via machine learning and entanglement renormalisation. Nature communications, 10(1):1-10, 2019.

51 Florian A. Y. N. Schröder, David H. P. Turban, Andrew J. Musser, Nicholas D. M. Hine, and Alex W. Chin. Tensor network simulation of multi-environmental open quantum dynamics via machine learning and entanglement renormalisation. Nature Communications, 10(1):1062, December 2019. ISSN 2041-1723. doi: 10.1038/s41467-019-09039-7. URL http://www . nature. com/articles/s41467-019-09039-7

52 Dvira Segal and Abraham Nitzan. Spin-Boson Thermal Rectifier. Physical Review Letters, 94(3):034301, January 2005. ISSN 0031-9007, 1079-7114. doi: 10.1103/PhysRevLett.94.034301. URL https://link. aps.org/doi/10.1103/PhysRevLett.94.034301.

53 Alejandro D. Somoza, Oliver Marty, James Lim, Susana F. Huelga, and Martin B. Plenio. Dissipation-assisted matrix product factorization. Phys. Rev. Lett., 123:100502, Sep 2019. doi: 10.1103/PhysRevLett.123.100502 URL https:// link.aps.org/doi/10.1103/PhysRevLett.123.100502

54 A. Strathearn, P. Kirton, D. Kilda, J. Keeling, and B. W. Lovett. Efficient non-Markovian quantum dynamics using time-evolving matrix product operators. $\mathrm{Na}$ ture Communications, 9(1):3322, December 2018. ISSN 2041-1723. doi:10.1038/s41467-018-05617-3 URL http: //www.nature.com/articles/s41467-018-05617-3.

55 Dario Tamascelli. Excitation Dynamics in Chain-Mapped Environments. Entropy, 22(11):1320, November 2020. doi:10.3390/e22111320 URL https://www.mdpi.com/ 1099-4300/22/11/1320. Number: 11 Publisher: Multidisciplinary Digital Publishing Institute.

56 Dario Tamascelli, Andrea Smirne, James Lim, Susana F. Huelga, and Martin B. Plenio. Efficient simulation of finite-temperature open quantum systems. Physical Review Letters, 123(9):090402, August 2019. ISSN 0031-9007, 1079-7114. doi: 
10.1103/PhysRevLett.123.090402 URL http://arxiv. org/abs/1811.12418 arXiv: 1811.12418.

or Maria Topaler and Nancy Makri. Quantum rates for a double well coupled to a dissipative bath: Accurate path integral results and comparison with approximate theories. The Journal of chemical physics, 101(9):7500-7519, 1994.

${ }^{58}$ H F Trotter. On the Product of Semi-Groups of Operators. Proceedings of the American Mathematical Society, 10(4):545-551, 1959.

59 Bram Vanhecke, Maarten Van Damme, Jutho Haegeman, Laurens Vanderstraeten, and Frank Verstraete. Tangent-space methods for truncating uniform MPS. arXiv:2001.11882 [cond-mat, physics:quant-ph], January 2020. URL http://arxiv.org/abs/2001.11882, arXiv: 2001.11882.

60 Haobin Wang and Jiushu Shao. Quantum phase tran- sition in the spin-boson model: A multilayer multiconfiguration time-dependent hartree study. The Journal of Physical Chemistry A, 123(9):1882-1893, 2019.

61 Ulrich Weiss. Quantum dissipative systems. World scientific, 2012.

${ }^{62}$ Mingru Yang and Steven R. White. Time-dependent variational principle with ancillary Krylov subspace. Physical Review B, 102(9):094315, September 2020. doi: 10.1103/PhysRevB.102.094315 URL https://link. aps.org/doi/10.1103/PhysRevB.102.094315. Publisher: American Physical Society.

63 Nengji Zhou, Lipeng Chen, Dazhi Xu, Vladimir Chernyak, and Yang Zhao. Symmetry and the critical phase of the two-bath spin-boson model: Groundstate properties. Physical Review B, 91(19):195129, May 2015. ISSN 1098-0121, 1550-235X. doi: 10.1103/PhysRevB.91.195129 URL https://link.aps . org/doi/10.1103/PhysRevB.91.195129 